\title{
114. DiSTRIBUCIÓN, ECOLOGÍA Y ESTADO DE CONSERVACIÓN DE TRAGOPOGON PSEUDOCASTELLANUS BLANCA \& DÍAZ DE LA GUARDIA (ASTERACEAE)
}

\author{
Félix LLAMAS, Carmen ACEDO y Raquel ALONSO REDONDO
}

Distribution, ecology and conservation status of Tragopogon pseudocastellanus Blanca \& Díaz de La Guardia (Asteraceae)

Palabras clave. Tragopogon, Asteraceae, conservación, fenología, ecología, León, España.

Key words. Tragopogon, Asteraceae, conservation, fenology, ecology, León, Spain.

La revisión de las especies ibéricas del género Tragopogon L. llevada a cabo por Blanca \& Díaz de La Guardia (1996) dio como resultado la descripción de una nueva especie, Tragopogon pseudocastellanus, mediante un pliego de herbario recolectado de forma casual años antes en la localidad de San Félix de Arce (Cabrillanes, León, España). Se trata de un taxon próximo a $T$. pratensis L., con lígulas amarillas e involucro con 5(7) brácteas, del que se diferencia por sus cipselas de mayor tamaño (20-25 mm frente a 17-22 en $T$. pratensis), hojas caulinares más anchas $(0,6$ $0,8 \mathrm{~cm}$ frente a 0,5 ó menos en $T$. pratensis) y lígulas de longitud $2 / 3$ de la de las brácteas siendo en $T$. pratensis de 4/5 o casi iguales a la longitud de las brácteas.

Hasta el momento no existía ninguna información sobre aspectos básicos de la biología y ecología de la especie, ni existían datos sobre la situación actual de este interesante microendemismo que figura con la categoría DD (Datos Insuficientes) en la Lista Roja de la Flora Vascular Española (Vv. Aa., 2000). Por esta razón, tratamos de dar a conocer la crítica situación actual de la especie, aportamos algunos datos básicos sobre su ecología y fenología, y señalamos medidas oportunas que puedan impedir la previsible desaparición de este taxon.

Distribución y ecología. La única población conocida hasta el momento es la de la localidad de la descripción original de San Félix de Arce (29TQH36), en el término municipal de Cabrillanes (León, España). No existe ninguna cita posterior de este taxon y nosotros sólo hemos podido encontrarlo en la misma localidad.

Desde el punto de vista biogeográfico, y atendiendo a la propuesta de Rivas-Martínez \& Loidi (1999), el territorio se encuadra en el subsector Ubiñense (sector UbiñensePicoeuropeano, subprovincia Orocantábrica, región Eurosiberiana) y esta localidad está situada en el piso supratemplado húmedo, con temperatura media anual de $7,6^{\circ} \mathrm{C}$ y precipitaciones anuales en torno a $1000 \mathrm{~mm}$.

Tragopogon pseudocastellanus vive en suelos ricos en bases de taludes terrosos con cierta nitrofilia, en orientaciones sur, a una altitud de unos $1230 \mathrm{~m}$, y en contacto con zonas de cultivos y prados de siega, al borde de la carretera. Fitosociológicamente, forma parte de comunidades incluidas en la clase Artemisietea vulgaris Lohmeyer, Preising \& Tüxen ex von Rochow, conviviendo con táxones como Achillea millefolium L., Convolvulus arvensis L., Ligusticum lucidum Miller, Papaver rhoeas L., Galium verum L., Daucus carota L., Centaurea nigra L., Eryngium campestre L., Dactylis glomerata L. y Sedum album $\mathrm{L}$. 


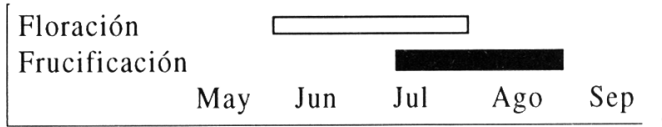

Figura 1. Fenología de Tragopogon pseudocastellanus. Fenology of Tragopogon pseudocastellanus.

Fenología. Según nuestras observaciones de campo y estimaciones, la floración de esta especie comienza en junio y finaliza hacia finales de julio. La aparición de los primeros frutos tiene lugar a mediados de julio y debe estar totalmente fructificada en agosto (fig. 1). Así, en el año 2001, a finales de julio estaban en fruto y diseminando las cipselas el $90 \%$ de la población, mientras que un $10 \%$ de los capítulos continuaba aún en floración.

Situación actual. En julio del año 2001, y como consecuencia de las actividades enmarcadas en el proyecto del Atlas de Flora Amenazada (AFA), realizamos un censo detallado de la única población conocida de Tragopogon pseudocastellanus mediante la técnica de marcaje y conteo directo, que arrojó un resultado de sólo 16 individuos adultos en un área de distribución de unos $50 \mathrm{~m}^{2}$. No hemos detectado la presencia de otras poblaciones en medios semejantes de las cuadrículas colindantes por lo que parece ser la única población existente. Por los resultados obtenidos (población única, número de individuos, área geográfica ocupada) podemos afirmar que la situación de esta especie en la actualidad es alarmante y debería ser catalogada como CR (en Peligro Crítico, por los criterios $\mathrm{A} 2 \mathrm{bc}, \mathrm{B} 1+2 \mathrm{abe}, \mathrm{C} 2 \mathrm{ab}, \mathrm{D}, \mathrm{E})$ y según las categorías UICN (2001). Como ya han señalado antes otros autores, las poblaciones pequeñas son particularmente propensas a extinguirse (Drury, 1974; Terborgh \& Winter, 1980; Shaffer, 1981; Primack, 1993).

Es necesario además señalar que el nivel de riesgo es elevado ya que la población se halla situada en un área de bastante actividad humana (actividad agrícola-ganadera, proximidad a una carretera y núcleos de población), por lo que el mantenimiento de su hábitat podría ser problemático. Otro problema añadido para la supervivencia de la población es la existencia de amenazas de origen biótico ya que según nuestras observaciones muchos de los capítulos tenían un porcentaje elevado de cipselas malogradas por causa del parasitismo por pulgones.

Estrategias de conservación. Aunque la información disponible es todavía insuficiente, desde el punto de vista de la protección de este endemismo, parece necesario adoptar las medidas adecuadas para evitar su extinción. Proponemos como medidas in situ el mantenimiento y vigilancia del hábitat, el seguimiento poblacional en los próximos años con el propósito de conocer si la población crece, decrece o se mantiene estable, y prospecciones en lugares ecológicamente semejantes para tratar de encontrar más poblaciones. Como medidas ex situ resultaría conveniente realizar estudios sobre la viabilidad de las semillas, y la germinación y supervivencia de las plántulas con vistas a su posterior reintroducción, y por supuesto, es urgente la inclusión de cipselas de Tragopogon pseudocastellanus en un banco de germoplasma solvente.

Otra propuesta adecuada para la conservación de la especie sería que el área en el que se sitúa la población de Tragopogon pseudocastellanus entrase a formar parte del Paisaje Protegido del Valle de San Emiliano, espacio de la REN (Red de Espacios Naturales de Castilla y León). Dicho espacio se halla muy próximo a la localidad de San Félix de Arce por lo que una ampliación con el propósito de acoger la población del endemismo que nos ocupa es perfectamente factible.

\section{BIBLIOGRAFÍA}

BLANCA, G. y C. DÍAZ DE LA GUARDIA -1996Sinopsis del género Tragopogon L. (Asteraceae) 
en la Península Ibérica. Anales Jard. Bot. Madrid 54: 358-363.

DRURY, W.H. -1974- Rare species. Biol. Conservation 6: 162-169.

PRIMACK, R.B. -1993- Essentials of conservation biology. Sinauer. Sunderland.

RIVAS-MARTÍNEZ, S. \& J. LOIDI - 1999 Byogeography of the Iberian Peninsula. Itinera Geobot. 13: 49-67.

SHAFFER, M.L. -1981- Minimum population sizes for species conservation. Bioscience 31: 131134.

TERBORGH, J. \& B. WINTER - 1980- Some causes of extinction. In: M.E. Soulé \& B.A. Wilcox (eds.). Conservation biology: an evolutionaryecological perspective: 119-133. Sinauer, Sunderland, Massachusetts.

UICN -2001- Categorías de listas rojas de la UICN: versión 3.1. Preparado por la Comisión de supervivencia de especies de la UICN. UICN, Gland, Suiza \& Cambridge, Reino Unido.

Vv. Aa. -2000- Lista Roja de la Flora Vascular Española. Conservación Vegetal 6: 1-40.

Aceptado para su publicación en julio de 2002

Dirección de los autores. F. LLAMAS GARCÍA y R. ALONSO REDONDO: Dpto. de Biología Vegetal (Botánica). Fac. de Ciencias Biológicas y Ambientales. Universidad de León. Campus de Vegazana s/n. 24071León (España). E-mail: dbvflg@unileon.es; dbvrar@unileon.es; C. ACEDO CASADO: Dpto. de Biología Vegetal (Botánica). Escuela Superior y Técnica de Ingeniería Agraria. Universidad de León. Campus de Ponferrada s/n. 24400-Ponferrada, León (España). E-niail: dbvcac@unileon.es

\title{
115. APORTACIONES A LA FLORA DE EXTREMADURA (ESPAÑA)
}

\author{
Francisco M. VÁZQUEZ PARDO, Antonio MARTÍN, \\ Soledad RAMOS MAQUEDA, Esperanza DONCEL PEREZ, \\ José BLANCO SALAS y Ana Belén LUCAS PIMIENTA.
}

Contributions of the Extremadura (Spain) flora

Palabras clave. Extremadura, Corología, Flora

Key words. Extremadura Corology, Flora.

Teline linifolia (L.) Webb

BADAJOZ: Garbayuela, bosque mixto de Quercus suber L. y Q. faginea Lam., 4-V-2001, E. Balbuena, E. Doncel, J. Pozo \& S. Ramos, HSIA 7274.

Especie que se encuentra acantonada en la mitad sur de España, en las Islas Baleares y en algunos puntos del Bajo Ampurdán (Talavera \&
Gibbs, 1999) en el norte de la Península Ibérica. En áreas limítrofes a la comunidad extremeña se conocía sólo en algunos puntos del norte de la provincia de Sevilla, (Alanís HSIA 2942) y ésta es la primera cita para la región extremeña, en áreas próximas a las poblaciones sevillanas anteriormente citadas. Se encuentra en áreas de alcornocales, donde aparecen dispersos pies de quejigos con un matorral 